\title{
Power variation \& stochastic volatility: a review and some new results
}

\author{
Ole E. BarndorfF-NiElsen \\ The Centre for Mathematical Physics and Stochastics (MaPhySto), \\ University of Aarhus, Ny Munkegade, DK-8000 Aarhus C, Denmark \\ oebn@imf .au.dk \\ SVEnd ERIK GRAVERSEN \\ Department of Mathematical Sciences, University of Aarhus, \\ Ny Munkegade, DK-8000 Aarhus C, Denmark \\ matseg@imf .au.dk \\ NEIL SHEPHARD \\ Nuffield College, University of Oxford, Oxford OX1 1NF, UK \\ neil.shephard@nuf .ox.ac.uk
}

First draft: January 2003. This draft May 2003

\begin{abstract}
In this paper we review some recent work on limit results on realised power variation, that is sums of powers of absolute increments of various semimartingales. A special case of this analysis is realised variance and its probability limit, quadratic variation. Such quantities often appear in financial econometrics in the analysis of volatility. The paper also provides some new results and discusses open issues.

Keywords: Bipower; Mixed Gaussian limit; Power variation; Quadratic variation; Realised variance; Realised volatility; Stochastic volatility.
\end{abstract}




\section{Contents}

1 Introduction $\quad 3$

2 Power variation: basic limit laws 4

$\begin{array}{lll}3 & \text { Stochastic volatility of unbounded variation } & 7\end{array}$

$\begin{array}{llr}4 & \text { Influence of leverage } & 8\end{array}$

5 Further extensions $\quad 9$

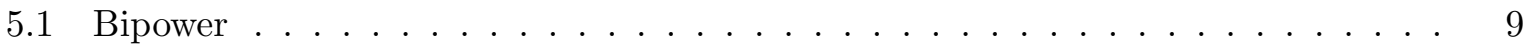

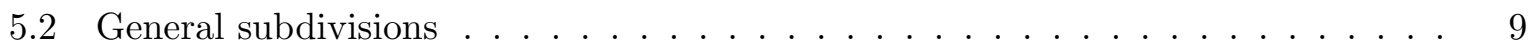

5.3 Weighted variations . . . . . . . . . . . . . . 10

5.4 Stable innovations . . . . . . . . . . . . . . . . . . . 10

5.5 Multivariate versions . . . . . . . . . . . . . . . . . 11

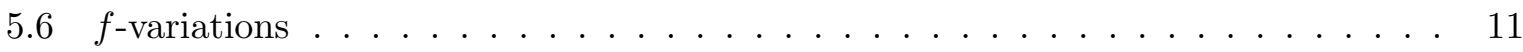

6 Conclusion $\quad 11$

7 Acknowledgements $\quad 11$ 


\section{Introduction}

The present paper provides an overview, including some new results and a discussion of open problems, of the theory and use of (realised) power variations. Most of the results to be discussed have been developed quite recently, as part of the quest to devise tools for more accurate assessments of stochastic volatility, particularly in the field of financial economics.

In that field a very important and broad class of models for the log price process of a financial asset is the following.

Definition 1 The class of stochastic volatility semimartingales (written $\mathcal{S} \mathcal{S} \mathcal{S}$ ) are those which can be written as $A+M$, where $A \in \mathcal{B V}$ (the class of processes of locally bounded variation) with $A(0)=0$ and $M \in \mathcal{M}_{l o c}$ (the class of local martingales) with $M(0)=0$, satisfying the additional condition that $M$ is a stochastic volatility $(\mathcal{S} \mathcal{V})$ process $M=H \cdot W$ where $W$ is standard Brownian motion and $H$, the so-called spot volatility, is càglàd and nonnegative. We also assume that $K^{*}(t)<\infty$ for all $t<\infty$, where

$$
K^{*}(t)=\int_{0}^{t} K(s) \mathrm{d} s
$$

and $K$, the so-called spot variance, is the squared volatility process, i.e. $K=H^{2}$. The class of continuous $\mathcal{S} \mathcal{V S M}$ is denoted by $\mathcal{S} \mathcal{V} \mathcal{S} \mathcal{M}^{c}$.

Note that our assumptions on $A$ and $H$ imply that these processes have locally Riemann integrable sample paths, while $M \in \mathcal{M}_{\text {loc }}^{c}$. Clearly $\mathcal{S} \mathcal{V} \mathcal{S} \in \mathcal{S} \mathcal{M}$ (the class of semimartingales). Overall if $X \in \mathcal{S} \mathcal{V} \mathcal{S M}$ then it can be written as

$$
X=A+H \bullet W .
$$

If we additionally impose the assumption that $A \in \mathcal{B V}^{c}$ then $X \in \mathcal{S} \mathcal{S} \mathcal{M}^{c} \in \mathcal{S M}^{c}$ (the class of continuous semimartingales). Note also that if $X \in \mathcal{S} \mathcal{V} \mathcal{S} \mathcal{M}^{c}$, then $X$ is a special semimartingale.

Reviews of the literature on stochastic volatility are given in Ghysels, Harvey, and Renault (1996) and Shephard (1996). By allowing the spot volatility $H$ to be random and serially dependent, this model will imply its increments will exhibit volatility clustering and have unconditional distributions which are fat tailed. This allows it to be used in finance and econometrics as a model for log-prices. In turn, this provides the basis for option pricing models which overcome some of the major failings in the Black-Scholes option pricing approach. Leading references in this regard include Hull and White (1987), Heston (1993) and Renault (1997). See also the recent work of Nicolato and Venardos (2002).

If $X \in \mathcal{S} \mathcal{V} \mathcal{S} \mathcal{M}^{c}$ then the quadratic variation $[X]$ of the process $X$ satisfies

$$
[X](t)=K^{*}(t)
$$

This quantity or other integrated powers of $H(t)$ are the key objects of study for this type of model. Econometric literature on the use of quadratic variation includes Andersen, Bollerslev, Diebold, and Labys (2003), Andersen, Bollerslev, and Diebold (2003), Barndorff-Nielsen and Shephard (2002a) and Barndorff-Nielsen and Shephard (2002b).

Section 2 recalls the concept of power variation and its basic probabilistic limit properties, which are subject to three main regularity assumptions. The roles of these assumptions in the proofs are outlined and then, in Sections 3 and 4 we briefly address the question of how the first two assumptions, that respectively prescribe a smoothness property of the sample paths of $H$ and stochastic independence of $H$ and $W$, may be relaxed. A number of other types of extension are reviewed in Section 5, and Section 6 concludes. 


\section{Power variation: basic limit laws}

Let $\Delta$ denote a subdivision $0=t_{0}<t_{1}<\cdots<t_{n}=t$ of $[0, t]$ and let $\delta_{j}=t_{j}-t_{j-1}$ and $|\Delta|=\max \delta_{j}$. For arbitrary real functions $f$ and $g$ on the interval $[0, t]$ we introduce the notation

$$
\left[f_{\Delta}\right]^{[r]}=\sum\left|f\left(t_{j}\right)-f\left(t_{j-1}\right)\right|^{r}
$$

where the sum is over $j=1, \ldots, n$. We call $\left[f_{\Delta}\right]^{[r]}$ the $r$-th order power variation of $f$ relative to $\Delta$. In the special case where the subdivision $\Delta$ is equidistant, whence $\delta_{j}=\delta$ for all $j$, we will write $f_{\delta}$ instead of $f_{\Delta}$, etc. Thus when $\delta$ occurs as an index the subdivision is understood to be equidistant.

Furthermore, when $f \geq 0$, we use the notation

$$
f^{*}(t)=\int_{0}^{t} f(s) \mathrm{d} s
$$

and, more generally,

$$
f^{r *}(t)=\int_{0}^{t} f^{r}(s) \mathrm{d} s .
$$

To formulate the basic limit laws we introduce the following four conditions.

(I) The processes $A$ and $H$ are jointly independent of $W$.

(V) The volatility process $H$ is (pathwise) locally bounded away from 0 and has, moreover, the property that for some $\gamma>0$

$$
\lim _{\delta \downarrow 0} \delta^{1 / 2} \sum_{j=1}^{n}\left|H^{\gamma}\left(\eta_{j}\right)-H^{\gamma}\left(\xi_{j}\right)\right|=0
$$

for any sequences $\xi_{j}=\xi_{j}(\delta)$ and $\eta_{j}=\eta_{j}(\delta)$ satisfying

$$
0 \leq \xi_{1} \leq \eta_{1} \leq \delta \leq \xi_{2} \leq \eta_{2} \leq 2 \delta \leq \cdots \leq \xi_{n} \leq \eta_{n} \leq t
$$

(M) The mean process $A$ satisfies (pathwise)

$$
\varlimsup_{\delta \downarrow 0} \max _{1 \leq j \leq n} \delta^{-1}|A(j \delta)-A((j-1) \delta)|<\infty .
$$

Remark 1 On account of the local Riemann integrability of $H$, the equality (5) is satisfied for all positive $\gamma$ if and only if it holds for one such $\gamma$.

In Barndorff-Nielsen and Shephard (2003a) the following result was proved (recall the notation (4)), extending an earlier result of Barndorff-Nielsen and Shephard (2002a) established in the special $r=2$ case.

Theorem 1 Let $X=A+H \bullet W$ be of class $\mathcal{S} \mathcal{S} \mathcal{S}$ and assume that the conditions (I), (V) and $(\mathrm{M})$ are satisfied.

Then $X \in \mathcal{S} \mathcal{V} \mathcal{S} \mathcal{M}^{c}$ and, for any $t>0$ and $\delta \downarrow 0$, we have

$$
\delta^{1-r / 2}\left[X_{\delta}\right]^{[r]}(t) \stackrel{p}{\rightarrow} \mu_{r} H^{r *}(t)
$$

where $\mu_{r}=\mathrm{E}\left\{|u|^{r}\right\}$ and $u \sim N(0,1)$. 
Furthermore,

$$
\frac{\delta^{1-r / 2}\left[X_{\delta}\right]^{[r]}(t)-\mu_{r} H^{r *}(t)}{\delta^{1-r / 2} \sqrt{\mu_{2 r}^{-1} v_{r}\left[X_{\delta}\right]^{[2 r]}(t)}} \stackrel{\text { law }}{\rightarrow} N(0,1),
$$

where $v_{r}=\operatorname{Var}\left\{|u|^{r}\right\}$ is the variance of $|u|^{r}$.

Remark 2 The conditions of Theorem 1 allow the volatility process $H$ to have, for example, deterministic diurnal effects, jumps, long memory, no unconditional mean or to be non-stationary.

In particular, we have that when $r=2$

$$
\frac{\left[X_{\delta}\right]^{[2]}(t)-H^{2 *}(t)}{\sqrt{\frac{2}{3}\left[X_{\delta}\right]^{[4]}(t)}} \stackrel{\text { law }}{\rightarrow} N(0,1) .
$$

Taking sums of squares of increments of log-prices has a very long tradition in financial economics — see, for example, Poterba and Summers (1986), Schwert (1989), Taylor and Xu (1997), Christensen and Prabhala (1998), Dacorogna, Müller, Olsen, and Pictet (1998), Andersen, Bollerslev, Diebold, and Labys (2001) and Andersen, Bollerslev, Diebold, and Ebens (2001). However, for a long time no theory was known for the behaviour of such sums outside the Brownian motion case. Since the link to quadratic variation has been made there has been a remarkably fast development in this field. Contributions include Andersen and Bollerslev (1998a), BarndorffNielsen and Shephard (2001) and Andersen, Bollerslev, Diebold, and Labys (2001). The limit result (8) was first given in Barndorff-Nielsen and Shephard (2002a).

When $r=1$ we have that

$$
\frac{\delta^{1 / 2}\left[X_{\delta}\right]^{[1]}(t)-\sqrt{2 / \pi} H^{*}(t)}{\sqrt{(1-2 / \pi) \delta\left[X_{\delta}\right]^{[2]}(t)}} \stackrel{\text { law }}{\longrightarrow} N(0,1) .
$$

Andersen and Bollerslev (1998b) and Andersen and Bollerslev (1997) empirically studied the properties of $\left[X_{\delta}\right]^{[1]}(t)$ computed using sums of absolute values of intra-day returns on speculative assets. This was empirically attractive, for using absolute values is less sensitive to possible large movements in high frequency data. There is evidence that if returns do not possess fourth moments then using absolute values rather than squares would be more reliable (see, for example, the work on the distributional behaviour of the correlogram of squared returns by Davis and Mikosch (1998) and Mikosch and Starica (2000)). However, the approach was abandoned in their subsequent work reported in Andersen and Bollerslev (1998a), and Andersen, Bollerslev, Diebold, and Labys (2001) due to the lack of appropriate theory for the sum of absolute returns as $\delta \downarrow 0$, although recently Andreou and Ghysels (2002) have performed some interesting Monte Carlo studies in this context, while Shiryaev (1999, pp. 349-350) and Maheswaran and Sims (1993) mention interests in the limit of sums of absolute returns. The above general limit theory, including the case $r=1$, was first given in Barndorff-Nielsen and Shephard (2003a).

Remark 3 Relation (7) may be rewritten as

$$
\frac{\delta^{1-r / 2} \mu_{r}^{-1}\left[X_{\delta}\right]^{[r]}(t)-H^{r *}(t)}{\delta^{1 / 2} \sqrt{\mu_{r}^{-2} \nu_{r} \mu_{2 r}^{-1} \delta^{1-r}\left[X_{\delta}\right]^{[2 r]}(t)}} \stackrel{\text { law }}{\rightarrow} N(0,1) .
$$

\footnotetext{
${ }^{1}$ In Barndorff-Nielsen and Shephard (2003a) the limit distribution result was stated under the additional condition that $r \geq \frac{1}{2}$ or $A=0$. However, as has kindly been pointed out to us by Jeanette Woerner, there is an error in the proof of the result given there, arising from an error in the inequality (22) of that paper. With a correct proof, see Barndorff-Nielsen and Shephard (2004), the additional condition is not needed.
} 
Here,

$$
\mu_{2 r}^{-1} \delta^{1-r}\left[X_{\delta}\right]^{[2 r]}(t) \stackrel{p}{\rightarrow} H^{2 r *}(t)
$$

so that

$$
\frac{\delta^{1-r / 2} \mu_{r}^{-1}\left[X_{\delta}\right]^{[r]}(t)-H^{r *}(t)}{\delta^{1 / 2} \sqrt{\mu_{r}^{-2} \nu_{r} H^{2 r *}(t)}} \stackrel{\text { law }}{\rightarrow} N(0,1) .
$$

In other words, $\delta^{1-r / 2} \mu_{r}^{-1}\left[X_{\delta}\right]^{[r]}(t)-H^{r *}(t)$ follows asymptotically a mixed normal distribution.

The following Sections discuss possible extensions of Theorem 1, in particular the question of weakening the conditions (I) and (V). Here, partly as a basis for the further discussion, we shall briefly indicate the main steps in the proof of the above results. For the rest of this Section we suppose that condition (I) is satisfied.

Recall that, by the Dambis-Dubins-Schwartz Theorem, any continuous local martingale $M$ is representable as $M=B(T)$ (a.s.) where $B$ is another Brownian motion and $T=[M]$. Thus $M$ has the form of a time-changed Brownian motion. In particular, we may rewrite (1) as

$$
X=A+B\left(K^{*}\right)
$$

a special case of the form

$$
X=A+B(T),
$$

where $T$ denotes a continuous increasing time change. It can be shown ${ }^{2}$ that under (I) not only are $K^{*}$ and $W$ independent but the same is true of $K^{*}$ and $B$.

For the time being let us consider the general form (11) with $T$ independent of $B$. Initially, we also assume that $A=0$. As above, let $\mu_{r}$ and $\nu_{r}$ denote the mean and variance of $|u|^{r}$ where $u$ is a standard normal variable. We then have that conditionally on $T$

$$
\left[X_{\delta}\right]^{[r]}(t)-\mu_{r}\left[T_{\delta}\right]^{[r / 2]}(t) \stackrel{\operatorname{law}}{=} \sum_{j=1}^{n} T_{\delta j}^{r / 2}\left(\left|u_{j}\right|^{r}-\mu_{r}\right),
$$

where $T_{\delta j}=T(\delta j)-T((j-1) \delta)$ and the $u_{j}$ are i.i.d. standard normal. This relation is the basis for the derivation of the subsequent limit law results. As the right hand side is a sum of independent random variables it is fairly clear that minor regularity requirements on $T$ will imply that

$$
\delta^{1-r / 2}\left(\left[X_{\delta}\right]^{[r]}(t)-\mu_{r}\left[T_{\delta}\right]^{[r / 2]}(t)\right) \stackrel{p}{\rightarrow} 0
$$

and

$$
\frac{\left[X_{\delta}\right]^{[r]}(t)-\mu_{r}\left[T_{\delta}\right]^{[r / 2]}(t)}{\sqrt{\nu_{r}\left[T_{\delta}\right]^{[r]}(t)}} \stackrel{\text { law }}{\rightarrow} N(0,1)
$$

and, combining the two previous relations,

$$
\frac{\delta^{1-r / 2}\left(\left[X_{\delta}\right]^{[r]}-\mu_{r}\left[T_{\delta}\right]^{[r / 2]}\right)}{\delta^{1 / 2} \sqrt{\mu_{2 r}^{-1} \nu_{r} \delta^{1-r}\left[X_{\delta}\right]^{[2 r]}}} \stackrel{\text { law }}{\longrightarrow} N(0,1) .
$$

Specifically, for (12) to hold it is sufficient that (a.s.) for $\delta \downarrow 0$

$$
\sup _{n} \delta^{1-r / 2} \sum_{j=1}^{n} T_{\delta j}^{r / 2}<\infty
$$

\footnotetext{
${ }^{2}$ See, for example, Barndorff-Nielsen and Shephard (2004, Ch. 4).
} 
while (13) is valid provided only

$$
\frac{\max _{j} T_{\delta j}^{r / 2}}{\sqrt{\left[T_{\delta}\right]^{[r]}(t)}} \rightarrow 0
$$

see Barndorff-Nielsen and Shephard (2003b). ${ }^{3}$

Now, when $T$ is of the form

$$
T=K^{*}(t)=\int_{0}^{t} K(s) \mathrm{d} s
$$

the Riemann integrability of $H$ implies

$$
\delta^{1-r / 2}\left[T_{\delta}\right]^{[r / 2]} \rightarrow \int_{0}^{t} K^{r / 2}(s) \mathrm{d} s=K^{r / 2 *}(t)=H^{r *}(t) .
$$

On account of (14) there is therefore reason to expect that, subject to a suitable regularity condition,

$$
\frac{\delta^{1-r / 2}\left[X_{\delta}\right]^{[r]}-\mu_{r} K^{r / 2 *}(t)}{\delta^{1 / 2} \sqrt{\mu_{2 r}^{-1} \nu_{r} \delta^{1-r}\left[X_{\delta}\right]^{[2 r]}}} \stackrel{\text { law }}{\longrightarrow} N(0,1)
$$

which is a statistically feasible result allowing us to draw inference about the volatility process $H$. The requirement for this latter conclusion to hold is that $\delta^{1-r / 2}\left[T_{\delta}\right]^{[r / 2]}-K^{r / 2 *}(t)$ tends to 0 sufficiently fast, specifically we need that

$$
\delta^{1-r / 2}\left[T_{\delta}\right]^{[r / 2]}-K^{r / 2 *}(t)=o\left(\delta^{1 / 2}\right) .
$$

In the quadratic variation case, i.e. $r=2$, this relation is trivially satisfied since $\left[T_{\delta}\right]^{[1]}=K^{*}(t)$. In general, condition ( $\mathbf{V})$ ensures the validity of (15), as is seen via the rewrite

$$
\delta^{1-r / 2}\left[T_{\delta}\right]^{[r / 2]}-K^{r / 2 *}(t)=\delta\left(\sum_{j=1}^{n}\left\{\left(\delta^{-1} \int_{(j-1) \delta}^{j \delta} H^{2}(s) \mathrm{d} s\right)^{r / 2}-\delta^{-1} \int_{(j-1) \delta}^{j \delta} H^{r}(s) \mathrm{d} s\right\}\right) .
$$

The final (and most intricate) step consists in showing that the main result (7) still holds if the process $A$ is not identically 0. Assumption (M) provides a necessary condition for this; for the proof, see Barndorff-Nielsen and Shephard (2004).

\section{Stochastic volatility of unbounded variation}

Condition (V) refers to each sample path of the volatility process individually. However, our main results concern limit distributions, for which a weaker condition could suffice. In fact, the following result holds.

Theorem 2 Let $X=H \bullet W$ with $H$ and $W$ independent and such that $H$ is nonnegative and has continuous sample paths. Fix $t>0$ and $r \geq 2$ and assume that, possibly under a locally equivalent probability measure,

(i) $H^{\rho}$ is a submartingale for all $\rho \geq 1$

(ii) $s \rightarrow \mathrm{E}\left\{H^{r}(s)\right\}$ belongs to $\operatorname{Lip}_{q}([0, t])$ for some $q>\frac{1}{2}$

\footnotetext{
${ }^{3}$ The conclusions (12), (13) and (14) could also, under slightly different conditions, be reached using general martingale limit theory as discussed in Hall and Heyde (1980; Section 3.2).
} 


$$
\frac{\delta^{1-r / 2}\left[X_{\delta}\right]^{[r]}(t)-\mu_{r} H^{r *}(t)}{\delta^{1-r / 2}\left\{\mu_{2 r}^{-1} v_{r}\left[X_{\delta}\right]^{[2 r]}(t)\right\}^{1 / 2}} \stackrel{\text { law }}{\rightarrow} N(0,1) .
$$

We conjecture that the result does, in fact, hold for all $r>0$ but have not been able to verify this. It appears likely that the theorem could, essentially, be obtained as a corollary from the extremely general theoretical framework discussed in an unpublished thesis by Becker (1998). However, in view of the generality and relative inaccessibility of that work is seems, in any case, desirable to have available a direct verification of Theorem 2 and we refer to Graversen (2003) for an independent proof. For a brief indication of the contents of Becker's thesis see Subsection 5.5 below.

With Theorem 2 at hand we can now, at least for $r \geq 2$, extend the validity of the conclusions of the original power variation Theorem to include for instance the square root (or Cox-IngersollRoss) process as model for the spot variance $K$.

\section{Influence of leverage}

In models encompassing leverage effects the volatility process (here $H$ ) and the innovation process (here $W$ ) will be correlated, such as is the case for the Heston (1993) model, for instance. Now, we know that under leverage we still have

$$
\delta^{1-r / 2}\left[X_{\delta}\right]^{[r]}(t) \stackrel{p}{\rightarrow} \mu_{r} H^{r *}(t),
$$

when $r=2$ through the usual quadratic variation result, while Woerner (2002) has extended the above convergence result to cover the case where $1<r<2$. Further, at first sight it seems plausible that the limit behaviour of

$$
\delta^{1-r / 2}\left[X_{\delta}\right]^{[r]}(t)-\mu_{r} H^{r *}(t)
$$

will still be mixed normal (cf. general martingale limit theory, see Hall and Heyde (1980, Section $3.2)$ ) whereas dependency between (16) and its asymptotic variance will prevent modification into a feasible standard normal limit as was done under the independence assumption in Section 2. However, the situation is even more intricate, as indicated by the following Theorem. There we consider the process

$$
X=|W|^{a} \bullet W,
$$

where $a \geq 1$. We have in mind ultimately to be able to handle more general cases, in particular that of the Heston model where $H$ is the square root, or CIR, process and is correlated with $W$. Again, it appears possible that this kind of setting is, at least in essence, covered by the general theory developed by Becker (1998, Subsection 5.5) but we have not been able to decide this and it is, moreover, illuminating to have a separate proof of the following Theorem; for such a proof, see Graversen (2003).

Theorem 3 For $t>0$ and $a \geq 1$ let

$$
X(t)=\int_{0}^{t}|W(s)|^{a} \mathrm{~d} W(s)
$$

(when a is a natural number the absolute sign may be dropped). Then

$$
\lim _{n}\left(\sqrt{n} \sum_{i=1}^{n}\left(X_{i t / n}-X_{(i-1) t / n}\right)^{2}-\int_{0}^{t}|W(s)|^{2 a} \mathrm{~d} s\right)
$$


exists in distribution for all $t>0$. The limit is mixed Gaussian. More precisely it is the distribution of

$$
\int_{0}^{t}|Y(s)|^{a} \mathrm{~d} Z(s)
$$

where $Y$ and $Z$ are two independent Wiener processes.

In relation to the martingale central limit theory one may ask whether the conclusions of the above Theorem follow from Hall and Heyde (1980, Theorem 3.2). However, that is not the case; specifically, neither of their conditions (3.19) and (3.21) are met in the present case.

\section{$5 \quad$ Further extensions}

This Section briefly reviews various further extensions of the basic results discussed in Section 2 .

\subsection{Bipower}

In Barndorff-Nielsen and Shephard (2003c) the concept of power variation is extended to bipower, and more generally multipower, variation. In Theorem 1 we considered the limiting behaviour of

$$
\delta^{1-r / 2}\left[X_{\delta}\right]^{[r]}(t)=\delta^{1-r / 2} \sum_{j=1}^{n}|X(j \delta)-X((j-1) \delta)|^{r} .
$$

The corresponding bipower quantity is defined as

$$
\left.\delta^{1-(r+s) / 2}\left[X_{\delta}\right]^{[r, s]}(t)=\delta^{1-(r+s) / 2} \sum_{j=1}^{n-1}|X(j \delta)-X((j-1) \delta)|^{r} \mid X((j+1) \delta)-X(j) \delta\right)\left.\right|^{s} .
$$

It has similar limit properties for $\mathcal{S} \mathcal{S} \mathcal{S M}^{c}$ models but generally differs when to a model of that kind is added a jump process. In particular, in case the jump intensity is not too high, $\left[X_{\delta}\right]^{[1,1]}(t)$ will still converge to the integrated squared volatility $K^{*}(t)$, and this allows separate estimation of the jump component of $[X](t)$.

\subsection{General subdivisions}

Let $\Delta$ denote a subdivision $0=t_{0}<t_{1}<\cdots<t_{n}=t$ of $[0, t]$ and let $\delta_{j}=t_{j}-t_{j-1}$ and $|\Delta|=\max \delta_{j}$. When considering a sequence of such subdivisions $\Delta$ we say that the sequence is balanced if $\max \delta_{j} / \min \delta_{j}$ is bounded above and $\varepsilon$-balanced, $\varepsilon \in(0,1)$, if $\max \delta_{j} /\left(\min \delta_{j}\right)^{\varepsilon} \rightarrow 0$ as $|\Delta| \rightarrow 0$. Note that here and in the following we have in mind a single, generally unspecified, sequence of subdivisions $\Delta$ with $|\Delta| \rightarrow 0$; however, for notational simplicity, we do not indicate this by attaching a sequence index to $\Delta$. Clearly, if $\Delta$ is balanced then it is a fortiori $\varepsilon$-balanced for every $\varepsilon \in(0,1)$.

Suppose again that $X$ is a process of the form $X=H \bullet W$. It is now convenient to introduce the notation

$$
{\overline{\left[X_{\Delta}\right]}}^{[r]}(t)=\sum \delta_{j}^{1-r / 2}\left|X\left(t_{j}\right)-X\left(t_{j-1}\right)\right|^{r}
$$

and the condition 
$(\overline{\mathbf{V}})$ The volatility process $H$ is (pathwise) bounded away from 0 and has, moreover, the property that for some $\gamma>0$ (equivalently for all $\gamma>0$ )

$$
\frac{\sum_{j=1}^{m} \delta_{j}\left|H^{\gamma}\left(\eta_{j}\right)-H^{\gamma}\left(\xi_{j}\right)\right|}{\sqrt{\min \delta_{j}}} \rightarrow 0
$$

for any sequences $\xi_{j}=\xi_{j}(\Delta)$ and $\eta_{j}=\eta_{j}(\Delta)$ satisfying

$$
0 \leq \xi_{1} \leq \eta_{1} \leq t_{1} \leq \xi_{2} \leq \eta_{2} \leq t_{2} \leq \cdots \leq \xi_{n} \leq \eta_{n} \leq t .
$$

In case $\Delta$ is equidistant condition $(\overline{\mathbf{V}})$ reduces to condition $(\mathbf{V})$.

Theorem 4 Let $X$ be a semimartingale of the form $X=H \bullet W$ and suppose that the volatility process $H$ is independent of the Brownian motion $W$ and satisfies conditions $(\mathbf{I})$ and $(\overline{\mathbf{V}})$. Then, for any $t>0$ and for any $\frac{1}{2}$-balanced sequence of subdivisions $\Delta$ we have

$$
{\overline{\left[X_{\Delta}\right]}}^{[r]}(t) \stackrel{p}{\rightarrow} \mu_{r} H^{r *}(t)
$$

as $|\Delta| \rightarrow 0$ and where $\mu_{r}=\mathrm{E}\left\{|u|^{r}\right\}$ and $u \sim N(0,1)$.

Furthermore, if the sequence of subdivisions $\Delta$ is $\frac{2}{3}$-balanced then

$$
\frac{{\overline{\left[X_{\Delta}\right.}}^{[r]}(t)-\mu_{r} H^{r *}(t)}{\sqrt{\mu_{2 r}^{-1} \nu_{r} \sum \delta_{j}^{2-r}\left|X\left(t_{j}\right)-X\left(t_{j-1}\right)\right|^{2 r}(t)}} \stackrel{\text { law }}{\rightarrow} N(0,1)
$$

where $\nu_{r}=\operatorname{Var}\left\{|u|^{r}\right\}$ is the variance of $|u|^{r}$.

The result is proved in Barndorff-Nielsen and Shephard (2003b). A recent paper by Woerner (2003) provides extensions of this, using techniques that are rather different from those of Barndorff-Nielsen and Shephard (2003b).

\subsection{Weighted variations}

For some applications it may be useful to generalise the above setup to allow for weighted power variations

$$
\left[f_{\Delta}\right]^{[F, r]}=\sum\left|f\left(t_{j}\right)-f\left(t_{j-1}\right)\right|^{r}\left(F\left(t_{j}\right)-F\left(t_{j-1}\right)\right) .
$$

where $F$ denotes a function of locally bounded variation. Under regularity restrictions one can show (cf. Barndorff-Nielsen and Shephard (2003b)) that, in notation similar to the above,

$$
\frac{\sum \delta_{j}^{-r / 2}\left|X_{j}\right|^{r} F_{j}-\mu_{r} \int_{0}^{t} H^{r}(s) \mathrm{d} F(s)}{\sqrt{2 \mu_{2 r}^{-1} \nu_{r} \sum \delta_{j}^{-r}\left|X_{j}\right|^{2 r} F_{j}^{2}}} \stackrel{\text { law }}{\rightarrow} N(0,1),
$$

where $F_{j}=F\left(t_{j}\right)-F\left(t_{j-1}\right)$.

\subsection{Stable innovations}

If the innovations come from a symmetric $\alpha$-stable motion $S$ (with $0<\alpha<2$ ) rather than from Brownian motion $W$, somewhat similar, but statistically less satisfactory, results can still be obtained, see Barndorff-Nielsen and Shephard (2003b). 


\subsection{Multivariate versions}

We are presently working on extensions of some of the above results to multivariate settings. For the case $r=2$, that is going from quadratic variation to quadratic covariation, this is rather straightforward, though for feasibility a special argument (related to the idea of bipower) is needed. This case is treated in considerable detail in Barndorff-Nielsen and Shephard (2002b).

\section{$5.6 f$-variations}

In a thesis from (1998) Etienne Becker has, in great depth, studied the limit behaviour as $M \rightarrow \infty$ of processes of the type

$$
Y_{M}(t)=\sum_{j=1}^{[M t]} f\left(\frac{j-1}{M}, \frac{1}{\sqrt{M}}\left\{X\left(\frac{j}{M}\right)\right\}-\left\{X\left(\frac{j-1}{M}\right)\right\}\right)
$$

where $f$ is a function of two variables and $X$ is a semimartingale. He considers in particular the case where $X$ is of the form

$$
X(t)=\int_{0}^{t} C(s) \mathrm{d} s+\int_{0}^{t} H(s) \mathrm{d} W(s),
$$

where $W$ is Brownian motion and $C$ and $H$ are predictable and subject to restrictions on their variational behaviour, and as a key result he proves that, after a suitable centering, $Y_{M}$ converges to a stochastic process which is representable as a certain type of stochastic integral where the integration is with respect to a 'martingale-measure tangential to $X$ '.

In comparing the results discussed in the previous sections to Becker's work we note that for the kind of functions, i.e. absolute powers, and regularity conditions we have considered more explicit results are possible. Thus, under assumptions (I), (V) and (M), the limit behaviour is identifiable as mixed Gaussian and random rescaling by observable scale factors leads to statistically directly applicable standard normal limit statements (cf. in particular Theorems 1 and 2). Moreover, for the special power variation setting of Section 4 it would seem possible to identify the 'tangential martingale-measure'. We hope, elsewhere, to discuss this latter point further.

\section{Conclusion}

In this paper we have reviewed and extended various recent results on realised power variation, a concept which appears useful in the context of changing volatility in financial economics. Key extensions are given to deal with unbounded variation in the volatility process and leverage.

It would be desirable to extend several of the results discussed above to functional limit theorems.

There are in the literature a considerable number of important results on power variations of semimartingales generally, and Lévy processes in particular, that are related but not directly relevant to what we have discussed above. A brief guide to those results are given in BarndorffNielsen and Shephard (2003b).

\section{Acknowledgements}

We would like to thank Jeannette Woerner for various conversations on this topic. Ole E. Barndorff-Nielsen's work is supported by CAF (www.caf.dk), which is funded by the Danish Social Science Research Council, and by MaPhySto (www.maphysto.dk), which is funded by 
the Danish National Research Foundation. Neil Shephard's research is supported by the UK's ESRC through the grants "Econometrics of trade-by-trade price dynamics" and "High frequency financial econometrics based upon power variation".

\section{References}

Andersen, T. G. and T. Bollerslev (1997). Intraday periodicity and volatility persistence in financial markets. Journal of Empirical Finance 4, 115-158.

Andersen, T. G. and T. Bollerslev (1998a). Answering the skeptics: yes, standard volatility models do provide accurate forecasts. International Economic Review 39, 885-905.

Andersen, T. G. and T. Bollerslev (1998b). Deutsche mark-dollar volatility: intraday activity patterns, macroeconomic announcements, and longer run dependencies. Journal of Finance 53, 219-265.

Andersen, T. G., T. Bollerslev, and F. X. Diebold (2003). Parametric and nonparametric measurement of volatility. In Y. Ait-Sahalia and L. P. Hansen (Eds.), Handbook of Financial Econometrics. Amsterdam: North Holland. Forthcoming.

Andersen, T. G., T. Bollerslev, F. X. Diebold, and H. Ebens (2001). The distribution of realized stock return volatility. Journal of Financial Economics 61, 43-76.

Andersen, T. G., T. Bollerslev, F. X. Diebold, and P. Labys (2001). The distribution of exchange rate volatility. Journal of the American Statistical Association 96, 42-55.

Andersen, T. G., T. Bollerslev, F. X. Diebold, and P. Labys (2003). Modeling and forecasting realized volatility. Econometrica 71, 579-625.

Andreou, E. and E. Ghysels (2002). Rolling-sampling volatility estimators: some new theoretical, simulation and empirical results. Journal of Business and Economic Statistics 20, $363-376$.

Barndorff-Nielsen, O. E. and N. Shephard (2001). Non-Gaussian Ornstein-Uhlenbeck-based models and some of their uses in financial economics (with discussion). Journal of the Royal Statistical Society, Series B 63, 167-241.

Barndorff-Nielsen, O. E. and N. Shephard (2002b). Econometric analysis of realised covariation: high frequency covariance, regression and correlation in financial economics. Unpublished paper: Nuffield College, Oxford, Economics working paper 2002-W13.

Barndorff-Nielsen, O. E. and N. Shephard (2002a). Econometric analysis of realised volatility and its use in estimating stochastic volatility models. Journal of the Royal Statistical Society, Series B 64, 253-280.

Barndorff-Nielsen, O. E. and N. Shephard (2003c). Power and bipower variation with stochastic volatility and jumps (with discussion). Journal of Financial Econometrics. Forthcoming.

Barndorff-Nielsen, O. E. and N. Shephard (2003b). Power variation and time change. Unpublished paper: Nuffield College, Oxford.

Barndorff-Nielsen, O. E. and N. Shephard (2003a). Realised power variation and stochastic volatility. Bernoulli 9, 243-265.

Barndorff-Nielsen, O. E. and N. Shephard (2004). Financial Volatility: Stochastic Volatility and Lévy based models. Cambridge: Cambridge University Press. Forthcoming.

Becker, E. (1998). Theorems limite pour des processus discretisés. Thése de Doctorat de l'Université Paris 6. 
Christensen, B. J. and N. R. Prabhala (1998). The relation between implied and realized volatility. Journal of Financial Economics 37, 125-150.

Dacorogna, M. M., U. A. Müller, R. B. Olsen, and O. V. Pictet (1998). Modelling short term volatility with GARCH and HARCH. In C. Dunis and B. Zhou (Eds.), Nonlinear Modelling of High Frequency Financial Time Series. Chichester: Wiley.

Davis, R. A. and T. Mikosch (1998). The limit theory for the sample ACF of stationary process with heavy tails with applications to ARCH. Annals of Statistics 26, 2049-2080.

Ghysels, E., A. C. Harvey, and E. Renault (1996). Stochastic volatility. In C. R. Rao and G. S. Maddala (Eds.), Statistical Methods in Finance, pp. 119-191. Amsterdam: North-Holland.

Graversen, S. E. (2003). Proofs of two theorems on power variation and stochastic volatility. Unpublished paper: Department of Mathematical Sciences, Aarhus University.

Hall, P. and C. C. Heyde (1980). Martingale Limit Theory and its Applications. San Diego: Academic Press.

Heston, S. L. (1993). A closed-form solution for options with stochastic volatility, with applications to bond and currency options. Review of Financial Studies 6, 327-343.

Hull, J. and A. White (1987). The pricing of options on assets with stochastic volatilities. Journal of Finance 42, 281-300.

Maheswaran, S. and C. A. Sims (1993). Empirical implications of arbitrage-free asset markets. In P. C. B. Phillips (Ed.), Models, Methods and Applications of Econometrics. Basil Blackwell.

Mikosch, T. and C. Starica (2000). Limit theory for the sample autocorrelations and extremes of a GARCH(1,1) process. Annals of Statistics 28, 1427-1451.

Nicolato, E. and E. Venardos (2002). Option pricing in stochastic volatility models of the Ornstein-Uhlenbeck type. Mathematical Finance. Forthcoming.

Poterba, J. and L. Summers (1986). The persistence of volatility and stock market fluctuations. American Economic Review 76, 1124-1141.

Renault, E. (1997). Econometric models of option pricing errors. In D. M. Kreps and K. F. Wallis (Eds.), Advances in Economics and Econometrics: Theory and Applications, pp. 223-78. Cambridge: Cambridge University Press.

Schwert, G. W. (1989). Why does stock market volatility change over time? Journal of Finance 44, 1115-1153.

Shephard, N. (1996). Statistical aspects of ARCH and stochastic volatility. In D. R. Cox, D. V. Hinkley, and O. E. Barndorff-Nielsen (Eds.), Time Series Models in Econometrics, Finance and Other Fields, pp. 1-67. London: Chapman \& Hall.

Shiryaev, A. N. (1999). Essentials of Stochastic Finance: Facts, Models and Theory. Singapore: World Scientific.

Taylor, S. J. and X. Xu (1997). The incremental volatility information in one million foreign exchange quotations. Journal of Empirical Finance 4, 317-340.

Woerner, J. (2002). Variational sums and power variation: a unifying approach to model selection and estimation in semimartingale models. Statistics and Decisions 21, 47-68.

Woerner, J. (2003). Estimation of integrated volatility in stochastic volatility models. Unpublished paper: OCIAM, Mathematical Institute, University of Oxford. 